\title{
Longitudinal Space Charge Amplifier
}

\author{
E. A. Schneidmiller and M.V. Yurkov \\ Deutsches Elektronen-Synchrotron (DESY), Notkestr. 85, D-22607 Hamburg, Germany
}

\begin{abstract}
Longitudinal space charge (LSC) driven microbunching instability in electron beam formation systems of X-ray FELs is a recently discovered effect hampering beam instrumentation and FEL operation. The instability was observed in different facilities in infrared and visible wavelength ranges. In this paper we propose to use such an instability for generation of vacuum ultraviolet (VUV) and X-ray radiation. A typical longitudinal space charge amplifier (LSCA) consists of few amplification cascades (drift space plus chicane) with a short undulator behind the last cascade. If the amplifier starts up from the shot noise, the amplified density modulation has a wide band, on the order of unity. The bandwidth of the radiation within the central cone is given by inverse number of undulator periods. A wavelength compression could be an attractive option for LSCA since the process is broadband, and a high compression stability is not required. LSCA can be used as a cheap addition to the existing or planned short-wavelength FELs. In particular, it can produce the second color for a pump-probe experiment. It is also possible to generate attosecond pulses in the VUV and X-ray regimes. Some user experiments can profit from a relatively large bandwidth of the radiation, and this is easy to obtain in LSCA scheme. Finally, since the amplification mechanism is broadband and robust, LSCA can be an interesting alternative to self-amplified spontaneous emission free electron laser (SASE FEL) in the case of using laser-plasma accelerators as drivers of light sources.
\end{abstract}

Keywords: Microbunching instability, space charge, X-ray

\section{INTRODUCTION}

Longitudinal space charge (LSC) driven microbunching instability ${ }^{1,2}$ in electron linacs with bunch compressors (used as drivers of short wavelength FELs) was a subject of intense theoretical and experimental studies during last years. ${ }^{3-9}$ Such instability develops in infrared and visible wavelength ranges and can hamper electron beam diagnostics and FEL operation.

In this paper we propose to use this effect for generation of VUV and X-ray radiation. We introduce a concept of a longitudinal space charge amplifier (LSCA) and present basic scaling relations in Section 2 . We discuss possible applications of such an amplifier in Section 3, and end up with discussion and conclusions in Section 4.

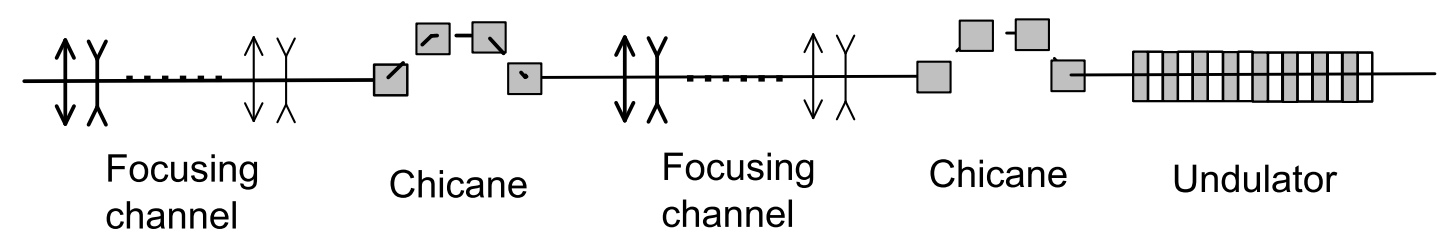

Figure 1. Conceptual scheme of an LSC amplifier. 


\section{GENERIC LSCA}

\subsection{Scheme of an LSCA}

Let us consider a scheme presented at Fig. 1. An amplification cascade consists of a focusing channel and a dispersive element (usually a chicane) with an optimized momentum compaction $R_{56}$. In a channel the energy modulations are accumulated, that are proportional to density modulations and space charge impedance of the drift space. In the chicane these energy modulations are converted into induced density modulations that are much larger that initial ones, ${ }^{1}$ the ratio defines a gain per cascade. In this paper we will mainly consider the case when the amplification starts up from the shot noise in the electron beam - although, in principle, the coherent density modulations can be amplified in the same way. A number of cascades is defined by the condition that the total gain, given by the product of partial gains in each cascade, is sufficient for saturation (density modulation on the order of unity) after the start up from shot noise. As we will see, in most cases two or three cascades would be sufficient. The amplified density modulation has a large relative bandwidth, typically in the range 50-100 \%. Behind the last cascade a radiator undulator is installed, which produces a powerful radiation with a relatively narrow line (inverse number of periods) within the central cone. This radiation is transversely coherent, and the longitudinal coherence length is given by the product of the number of undulator periods by the radiation wavelength. When LSCA saturates in the last cascade, a typical enhancement of the radiation intensity over that of spontaneous emission is given by a number of electrons per wavelength.

\subsection{Formula for a gain per cascade}

Let us now present simple formulas for calculations of the gain and optimization of parameters of an LSCA. As in the case of a SASE FEL, ${ }^{10}$ we assume that at the entrance to the amplifier there is only shot noise in the electron beam. Let us consider the linear amplification of spectral components of the noise within the amplifier band. The formula for amplitude gain per cascade was obtained in studies of microbunching instabilities in linacs with bunch compressors: ${ }^{1}$

$$
G_{n}=C k\left|R_{56}\right| \frac{I}{\gamma I_{\mathrm{A}}} \frac{4 \pi|Z(k)| L_{d}}{Z_{0}} \exp \left(-\frac{1}{2} C^{2} k^{2} R_{56}^{2} \frac{\sigma_{\gamma}^{2}}{\gamma^{2}}\right)
$$

Here $k=1 / \lambda=2 \pi / \lambda$ is the modulation wavenumber, $Z$ is the impedance of a drift space (per unit length), $Z_{0}$ is the free-space impedance, $L_{d}$ is the length of the drift space, $I$ is the beam current, $I_{\mathrm{A}}$ is the Alfven current, $R_{56}$ is the compaction factor of a chicane, $C$ is the compression factor*, $\gamma$ is relativistic factor, and $\sigma_{\gamma}$ is rms uncorrelated energy spread (in units of rest energy). It is assumed here that energy modulations are accumulated upstream of the chicane but not inside: there the self-interaction is suppressed due to $R_{51}$ and $R_{52}$ effects. ${ }^{11-13}$ We also assume in the following that a length of the drift space is much larger than that of the chicane, while the $R_{56}$ of the drift space is much smaller. Also note that the formula (1) was obtained under the condition of high gain, $G \gg 1$. In this case both the sign of $R_{56}$ and the phase of impedance are not important. The value of the $R_{56}$ is to be optimized for a highest gain at a desired wavelength.

In this paper we will consider LSC induced energy modulations in a drift space or in an undulator (at a wavelength that is much longer than the resonant one). In the latter case ${ }^{\dagger}$ the relativistic factor $\gamma$ in the formulas for impedance should be substituted by the longitudinal relativistic factor $\gamma_{z}:{ }^{14}$

$$
\frac{4 \pi Z(k)}{Z_{0}}=\frac{2 i k}{\gamma_{z}^{2}} \int d r_{\perp}^{\prime} \int d r_{\perp}^{\overrightarrow{\prime \prime}} \rho\left(\overrightarrow{r_{\perp}^{\prime}}\right) \rho\left(\overrightarrow{r_{\perp}^{\prime \prime}}\right) K_{0}\left(\frac{k\left|\overrightarrow{r_{\perp}^{\prime}}-\overrightarrow{r_{\perp}^{\prime \prime}}\right|}{\gamma_{z}}\right)
$$

Here $\rho(\vec{\perp})$ is the transverse distribution of the current density and $K_{0}$ is the modified Bessel function. In particular, in the case of a pencil beam limit for the space charge,

\footnotetext{
${ }^{*}$ The wavenumber after the chicane is $C k$.

${ }^{\dagger}$ Under the condition $\sigma_{\perp}^{2} \ll \lambda \lambda_{w}$, where $\lambda_{w}=\lambda_{w} / 2 \pi$ is the reduced undulator period and $\sigma_{\perp}$ is rms transverse size of the beam.
} 


$$
\lambda \gamma_{z} \gg \sigma_{\perp}
$$

$\sigma_{\perp}$ being an rms transverse size of the beam, the LSC impedance is given by

$$
\frac{4 \pi Z(k)}{Z_{0}}=\frac{i k}{\gamma_{z}^{2}}\left(2 \ln \frac{\gamma_{z}}{k \sigma_{\perp}}+\text { const. }\right)
$$

Here the constant in the brackets depends on transverse shape of the beam.

We request that a density modulation does not change significantly in the drift space. This may happen due to plasma oscillations or due to a spread of longitudinal velocities for a finite beam emittance. Thus, a drift space length is limited by the condition:

$$
L_{d} \leq \min \left(L_{1}, L_{2}\right)
$$

Here $L_{1}$ is the reduced wavelength $\lambda_{p}$ of plasma oscillations:

$$
L_{1} \simeq \lambda_{p}=\gamma_{z}\left(\frac{I}{\gamma I_{\mathrm{A}}} \frac{4 \pi|Z| k}{Z_{0}}\right)^{-1 / 2}
$$

The second limitation follows from the condition that the longitudinal velocity spread due to a finite beam emittance does not spoil the modulations during the passage of the drift:

$$
L_{2} \simeq \frac{\lambda}{\sigma_{\theta}^{2}}=\frac{\beta \lambda}{\epsilon},
$$

where $\sigma_{\theta}$ is the angular spread in the beam, $\beta$ is the beta function, $\epsilon=\epsilon_{n} / \gamma$ is beam emittance, $\epsilon_{n}$ is the normalized emittance.

\subsection{Formulas for an optimized LSC amplifier}

Let us first consider the case without wavelength compression, $C=1$. We start optimization assuming that the beam parameters are fixed: current $I$, normalized emittance $\epsilon_{n}$, beam energy $\gamma$ and energy spread $\sigma_{\gamma}$ in units of the rest energy, and longitudinal gamma-factor $\gamma_{z}$. We can select a central wavelength, optimize $R_{56}$ of the dispersion section for the chosen wavelength, choose beta-function and optimize a length of the drift space. Our goal is to get a highest gain at a shortest wavelength.

The impedance increases with $k$, achieves the maximum at

$$
\lambda \simeq \lambda_{o p t} \simeq \frac{\sigma_{\perp}}{\gamma_{z}}=\frac{\sqrt{\epsilon \beta}}{\gamma_{z}},
$$

and then decays in the asymptote of a pancake beam. Let us consider the wavelength about $2 \pi \lambda_{\text {opt }}$ as an optimum choice, since the impedance is the largest, and transverse correlations of the LSC field are still on the order of the beam size. The impedance at this wavelength can be approximated by

$$
\frac{4 \pi|Z|}{Z_{0}} \simeq \frac{1}{\lambda \gamma_{z}^{2}} \simeq \frac{1}{\sigma_{\perp} \gamma_{z}}
$$

It is slightly underestimated, but we ignore here numerical factors on the order of unity. Note that the impedance is rather flat around the optimal wavelength, it reduces by $10-15 \%$ when the wavelength is by a factor of 2 smaller or larger than the optimal one. The optimal $R_{56}$ of the dispersion section is

$$
R_{56} \simeq \lambda \frac{\gamma}{\sigma_{\gamma}}
$$


Substituting (9) and (10) into (1), we get an estimate of the amplitude gain per cascade for the wavelength given by $(8)$ :

$$
G_{n} \simeq \frac{I}{\sigma_{\gamma} I_{\mathrm{A}}} \frac{L_{d}}{\lambda \gamma_{z}^{2}} .
$$

Thus, the gain per cascade is approximately equal to the longitudinal brightness of the electron beam multiplied by a number of LSC formation lengths.

Let us now consider the limitations on the drift length (5). The first limit (6) can be rewritten with the help of (9) as:

$$
L_{1} \simeq \lambda_{p} \simeq \lambda \gamma_{z}^{2} \sqrt{\frac{\gamma I_{\mathrm{A}}}{I}} .
$$

So, in the case $L_{1}<L_{2}$ the drift space length can be chosen to be $L_{d} \simeq L_{1}$. In this case the expression for the gain (11) reduces to:

$$
G_{n} \simeq \frac{1}{\sigma_{\gamma}} \sqrt{\frac{\gamma I}{I_{\mathrm{A}}}} .
$$

This estimate for the gain was originally obtained in. ${ }^{1}$ In the considered limit we have a relatively large betafunction. It is advisable to reduce it (if technically possible), since the wavelength (8) and length of the drift space (12) are also reduced but the gain (13) stays the same. This happens until the spread of longitudinal velocities starts playing a role, i.e. when $L_{1} \simeq L_{2}$. The corresponding beta-function is

$$
\beta_{c r} \simeq \epsilon \gamma_{z}^{2} \sqrt{\frac{\gamma I_{\mathrm{A}}}{I}} .
$$

If one further reduces beta-function, $\beta<\beta_{c r}$, the maximal drift length is given by $L_{d} \simeq L_{2}$. In this case from $(7),(8)$, and (11) we find that the gain is proportional to the beam brightness in 6 -D phase space:

$$
G_{n} \simeq \frac{I}{\sigma_{\gamma} I_{\mathrm{A}}} \frac{\beta}{\epsilon \gamma_{z}^{2}} \simeq \frac{I}{\sigma_{\gamma} I_{\mathrm{A}}}\left(\frac{\lambda}{\epsilon}\right)^{2} .
$$

Although the gain can still be high for $\lambda \gg \epsilon$, it quickly decreases when one goes to shorter wavelengths contrary to the case (13). Thus, the condition $L_{d} \simeq L_{1} \simeq L_{2}$ (and $\beta \simeq \beta_{c r}$ ) allows one to get the highest gain at the shortest wavelength. At this point we have:

$$
\begin{gathered}
\lambda \simeq \epsilon\left(\frac{\gamma I_{\mathrm{A}}}{I}\right)^{1 / 4}, \\
L_{d} \simeq \epsilon \gamma_{z}^{2}\left(\frac{\gamma I_{\mathrm{A}}}{I}\right)^{3 / 4} .
\end{gathered}
$$

The amplitude gain per cascade is given by (13), the beta function is given by (14), and the $R_{56}$ is given by (10).

To estimate the total gain (and number of cascades) required to reach saturation in LSCA, one has to estimate typical density modulation for the start-up from shot noise. The power spectral gain of the amplifier depends on the number of cascades $n$. For an optimal wavelength (8) as a central wavelength ( neglecting the dependence of the impedance on $k$ ), and for the optimized $R_{56}$ from (10), one easily obtains from (1) that the total power gain is proportional to $\hat{k}^{2 n} \exp \left(-n \hat{k}^{2}\right)$, where $\hat{k}=k / k_{\text {opt }}$. Thus, the relative bandwidth of the amplifier is in the 
range 50-100 \%, depending on the number of cascades. Then an effective shot noise density modulation can be estimated as: ${ }^{11}$

$$
\rho_{s h} \simeq \frac{1}{\sqrt{N_{\lambda}}}
$$

where $N_{\lambda}=I \lambda /(e c)$ is a number of electrons per central wavelength of the amplified spectrum, $\lambda=2 \pi \lambda$. At saturation the density modulation $\rho_{\text {sat }}$ is on the order of unity, so that the total amplitude gain $\rho_{\text {sat }} / \rho_{\text {sh }}$ is:

$$
G_{t o t}=G_{1} G_{2} \ldots G_{n} \simeq \sqrt{N_{\lambda}}
$$

where $G_{n}$ is the gain in the $n$-th cascade. The total power gain of the saturated amplifier (that shows an enhancement of power in a radiator with respect to spontaneous emission) is simply:

$$
G_{t o t}^{(p)}=G_{t o t}^{2} \simeq N_{\lambda}
$$

We have presented a simple scheme for optimization of LSCA, but we should note that it is not strict and serves for orientation in the parameter space only. For instance, one can choose a drift length that is significantly shorter than the limit given by (5) and increase the number of cascades instead. In this case the formula (11) should be used to calculate the gain. For instance, three cascades with the gain 10 in each give about the same total gain as two cascades with the gain 30 in each, but the total length of the amplifier (for the same betafunction) can be almost twice shorter in the former case. If in addition one reduces beta-function (since the drift space got shorter), one can go to shorter wavelength and higher gain per cascade. So, if the gain per cascade at $\beta \simeq \beta_{c r}$ is very large, it could be beneficial to go to the limit $L_{d} \simeq L_{2}<L_{1}$ - if the beta-function is not getting too low for technical realization. On the other hand, in many cases even beta-function given by (14) is too small and technically not feasible. In that case one would have to use larger values of $\beta$ and go to the limit defined by plasma oscillations only, thus using Eqs. (8)-(13). If the wavelength of interest differs significantly from the one given by (8), one should use more general formulas of the previous Section. In any case, the formulas of this Section are only estimates, and for more accurate gain calculations one should use more general formulas of the previous Section (but then one has to specify distributions), and in addition to include dynamics in drifts and chicanes more accurately.

\subsection{Wavelength compression}

As one can see from the formulas of the optimized LSCA, a typical operating wavelength of an optimized LSCA is significantly longer than a wavelength that can be reached in SASE FELs (they can lase at $\lambda \simeq \epsilon$ ). In order to go to shorter wavelengths for given electron beam parameters in LSCA, one would have to use wavelength compression. The broadband nature of the amplifier makes this option especially attractive. Indeed, the compression factor is given by the formula:

$$
C=\left(1-h R_{56}\right)^{-1}
$$

where $h$ is the linear energy chirp (the derivative of relative energy deviation). For a large $C$ a variation of the compression factor reads:

$$
\frac{\Delta C}{C} \simeq C \frac{\Delta h}{h}
$$

After the compression the bands of density modulations and of the radiator must overlap. This leads to the following requirement on the compression stability:

$$
\frac{\Delta C}{C}<\frac{\Delta k_{\max }}{k}
$$


where $\Delta k_{\max }=\max \left(\Delta k_{d e n}, \Delta k_{\text {rad }}\right)$, and $\Delta k_{d e n}$ and $\Delta k_{\text {rad }}$ are bandwidths of the density modulation and of the radiator, respectively. Thus, the stability of the chirp must satisfy the requirement:

$$
\frac{\Delta h}{h}<\frac{1}{C} \frac{\Delta k_{\max }}{k} .
$$

For coherent FEL-type modulations and an undulator as a radiator $\Delta k_{\max } / k \ll 1$ what might set very tight tolerance for the chirp stability and limit practically achievable compression factors. For an LSCA, however, $\Delta k_{\text {max }} / k=\Delta k_{\text {den }} / k \simeq 1$, so that for a given chirp stability one can go for much larger compression. Alternatively, for a given compression factor one can significantly loosen the tolerances. Note also that nonlinearities of the longitudinal phase space do not play a significant role in the case of LSCA.

If the wavelength compression is applied, one should use formula (1) to calculate gain per cascade, and adjust $R_{56}$ to optimize the gain depending on compression factor $C$. One can consider different options for compression. One possibility is to create an energy chirp before beam enters LSCA. In this case one can adjust $R_{56}$ in different cascades in order to have a mild compression in each cascade - but this might shift the wavelength beyond the optimal range in the drifts of last cascades. In that case one should make sure that the number of cascades and their parameters are adjusted such that a saturation of LSCA is finally achieved. Another possibility is to create an energy chirp before the last cascade (for instance, by a laser in a short undulator ${ }^{26}$ ), and get the desired compression in the last chicane. In this case, perhaps, one would need very strong energy chirp. An interesting option for a short electron bunch with a high current would be to use an energy chirp, induced by LSC along the whole bunch (then for compression one should use, for instance, doglegs instead of chicanes, taking into account the sign of the energy chirp). In this case one should carefully adjust parameters of the amplifier cascades since LSC (and a chirp) might strongly increase from one cascade to the next one. However, loose tolerances (24) can make such an option feasible.

\subsection{Undulator}

At the entrance of the undulator we have chaotically modulated electron beam with a typical amplitude of the order of unity at saturation. The temporal correlations have the scale of a wavelength, and the spectrum is broad. The undulator radiation within the central cone $\ddagger \sqrt{\lambda / L_{w}}$ (here $L_{w}$ is the undulator length) has a relative bandwidth $N_{w}^{-1} \ll 1$, where $N_{w}$ is the number of undulator periods. In the case when Fresnel number is small, $N_{f}=\sigma_{\perp}^{2} /\left(\lambda L_{w}\right) \ll 1$, the radiation power within the central cone is equal to the power of spontaneous emission (about 0.011 photons per electron are radiated within the central cone of a planar undulator with a large undulator parameter K) multiplied by the power gain $N_{\lambda}$ of the LSCA at saturation:

$$
W \simeq W_{s p} N_{\lambda} .
$$

In this limit the power within the central cone does not depend on the number of undulator periods. One can easily see that the Fresnel number is always small if the condition (8) is satisfied, transverse size of the beam in the undulator is the same as that in amplification cascades, and there is no wavelength compression. In this case the transverse coherence is guaranteed. However, with a strong wavelength compression in the last chicane of LSCA, the Fresnel number might no longer be small, so that one should use a more general formula. For Gaussian transverse distribution of the beam density, and under assumption that the amplified density modulations are transversely correlated, we can use the following expression for the power:

$$
W \simeq W_{s p} N_{\lambda} F\left(N_{f}\right)
$$

where the suppression factor is: ${ }^{15}$

$$
F\left(N_{f}\right)=\frac{2}{\pi}\left[\arctan \left(\frac{1}{2 N_{f}}\right)+N_{f} \ln \left(\frac{4 N_{f}^{2}}{4 N_{f}^{2}+1}\right)\right] .
$$

\footnotetext{
${ }^{\ddagger}$ There is, in general, also emission of powerful radiation beyond the central cone.
} 
For a large Fresnel number the last expression reduces to $F\left(N_{f}\right) \simeq 1 /\left(2 \pi N_{f}\right)$, and the power (26) is emitted within the angle $\lambda / \sigma_{\perp}$ which is much smaller than the central cone. In that case we still assume a relatively good transverse coherence if in the drifts of LSCA the wavelength is given by the condition (8) or it is longer. Indeed, transverse correlations are established in this case due to LSC. ${ }^{4}$ Longitudinal coherence length, as in the case of spontaneous undulator radiation, is given by the product of the wavelength and the number of undulator periods. We do not discuss in this paper nonlinear harmonic generation in LSCA, since it would be highly speculative without numerical simulations. We can only mention here that this should be possible in a saturated LSCA.

\section{POSSIBLE APPLICATIONS OF LSCA}

\subsection{LSCA as a cheap addition to existing or planned X-ray FELs}

Undulator beamlines of the existing and planned X-ray FELs often consist of long drift spaces and long undulators. Insertion of a few chicanes and a short undulator at the end may allow for a parasitic production of relatively long wavelength radiation (as compared with the FEL wavelength) by the same electron bunch. This would extend in an inexpensive way the wavelength range of a facility. Moreover, since both radiation pulses are perfectly synchronized, they can be used in pump-probe experiments.

As a first example let us consider the undulator beamline SASE1 at the European XFEL. ${ }^{16}$ There is a long drift space (about $300 \mathrm{~m}$ ) in front of SASE1 undulator, and $200 \mathrm{~m}$ long drift behind the undulator. The undulator itself has the total length of $200 \mathrm{~m}$ (magnetic length $165 \mathrm{~m}$ plus 35 meters of intersections). Let us consider the electron beam with the following parameters: energy $17.5 \mathrm{GeV}$, normalized slice emittance $0.4 \mathrm{~mm}$ mrad, peak current 3-4 kA, slice energy spread $1.5 \mathrm{MeV}$. The tunable-gap undulator is assumed to be tuned to the resonance with the wavelength $0.05 \mathrm{~nm}$, so that $\gamma_{z}=1.9 \times 10^{4}$. The optimal beta-function in the undulator for these beam parameters is about $15 \mathrm{~m}$, and it is about $30-40 \mathrm{~m}$ in the drifts. The core of the bunch with high current saturates at the FEL wavelength in the undulator, so that this part of the bunch is spoiled (has a large energy spread). We consider parts of the bunch with the current about $1 \mathrm{kA}$ assuming that there is no FEL saturation there. We propose to install three compact chicanes just in front of the undulator, just behind it, and at the end of the second drift. Thus, we have three amplification cascades of LSCA that operates parasitically. The last chicane is followed by a short undulator. From the formulas of the previous Section we find that the optimal wavelength for LSC instability is $\lambda \simeq 4 \mathrm{~nm}$. The optimal $R_{56}$ is about $8 \mu \mathrm{m}$ for all cacsades. Beta-function in all cascades is much larger than $\beta_{c r}$, moreover the lengths of all cascades are shorter than reduced wavelength of plasma oscillations, i.e. $L_{d}<L_{1}$. Therefore, we use formula (11) to calculate gain in every cascade. We find that the total gain is given by the following product of partial gains: $G_{t o t} \simeq 8 \times 13 \times 5 \simeq 500$. This is larger than the gain required to reach saturation, about 300 according to (19). We choose an undulator with 50 periods and a period length $10 \mathrm{~cm}$. Radiation power within the central cone exceeds that of spontaneous emission by 5 orders of magnitude and is in sub-GW level with the bandwidth about $2 \%$, radiation is transversely coherent. The tunability can be easily achieved in the range of 2-10 $\mathrm{nm}$ by changing the $R_{56}$ and the undulator gap. The soft X-ray pulses are synchronized with hard X-ray pulses produced by the core of the same bunch, so that these two colors can be used in pump-probe experiments. Alternatively, they can be separated and used by different experiments ${ }^{\S}$.

Note that in this example we considered a parasitic use of the beamline and of an unspoiled part of the electron bunch. With a dedicated use of the high-current part of the bunch one can essentially reduce the total length of the amplifier. Let us consider the same electron bunch as before, but now we assume that the core of the bunch with the current $3 \mathrm{kA}$ is not spoiled by FEL interaction (for instance, some bunches are kicked in front of the undulator by the fast kicker ${ }^{17}$ ). We consider an operation of LSCA in the drift behind the undulator, requiring beta-function to be about $10 \mathrm{~m}$ (somewhat larger that $\beta_{c r}$ ), thus the optimal wavelength is $2 \mathrm{~nm}$. Choosing length of the drift in an amplification cascade to be $30 \mathrm{~m}$ (it is much smaller than $\lambda_{p}$ ), and the $R_{56} \simeq 4 \mu \mathrm{m}$, we find with the help of (11) that the gain per cascade is about 5 . To reach saturation one would need four cascades, so that the total length of the amplifier would be about $120 \mathrm{~m}$. A gigawatt-level radiation

\footnotetext{
${ }^{\S}$ As an option one can consider the bending system (with properly adjusted $R_{56}$ ) between SASE1 and the downstream soft X-ray undulator SASE3 ${ }^{16}$ as an alternative to the last chicane. Then the short undulator is placed in SASE3 beamline thus extending its wavelength range.
} 
power would then be produced within the central cone of a short undulator, tunability between $1 \mathrm{~nm}$ and $5 \mathrm{~nm}$ is easy to obtain.

Parasitic use of long drifts and unspoiled parts of an electron beam is possible at other facilities, for example, at the soft X-ray FEL user facility FLASH. ${ }^{18,19}$ There is about $45 \mathrm{~m}$ long drift space in front of the $27 \mathrm{~m}$ long undulator. Without going into the details, we notice that by installing two chicanes (in front of the undulator and behind it, $R_{56} \simeq 200 \mu \mathrm{m}$ ) and a short radiator undulator one can parasitically generate powerful VUV radiation with the wavelength around $100 \mathrm{~nm}$.

\subsection{Generation of attosecond pulses}

There are many proposals to produce attosecond pulses from FELs. ${ }^{20-26}$ In principle, by using strongly nonlinear manipulations with the longitudinal phase space, one can reduce X-ray pulse duration down to several cycles. ${ }^{26}$ Here we note that the broadband nature of the LSC instability suggests that few-cycle pulses can be naturally produced in LSCA.

As an example, let us consider the electron beam with the following parameters: the energy $1.5 \mathrm{GeV}$, bunch charge $200 \mathrm{pC}$, peak current $1 \mathrm{kA}$, normalized emittance $0.5 \mu \mathrm{m}$, rms energy spread $100 \mathrm{keV}\left(\sigma_{\gamma}=0.2\right)$. We choose beta-function to be $1.5 \mathrm{~m}$, i.e. much larger than the value one gets from (14). From (16) we get $\lambda \simeq 5$ $\mathrm{nm}$, i.e. the wavelength $\lambda$ is about $30 \mathrm{~nm}$. The optimal $R_{56}$ for this wavelength is about $75 \mu \mathrm{m}$, the chicane can be as short as $50 \mathrm{~cm}$. We choose the length of a drift space $L_{d}=1.5 \mathrm{~m}$ which is much smaller than that given by the formula (17). One can find from (13) that the amplitude gain per cascade is about 10, i.e. we need three cascades to reach saturation according to (19).

Now let us introduce wavelength compression into the scheme. This can be done in a way similar to that considered in. ${ }^{26}$ In front of the last chicane we install a two-period undulator (period length $10 \mathrm{~cm}$ ) which is resonant with the wavelength of $750 \mathrm{~nm}$ of a laser producing pulses with energy of $1 \mathrm{~mJ}$ and duration of 5 fs (FWHM). The maximum of energy modulation can be about $15 \mathrm{MeV}$ and should be adjusted for a desired compression. Note that one can in-couple the laser beam at the position of the previous chicane where the electron beam deviates from the axis by a few millimeters. Consider as an example the compression of a short slice, having the maximal energy slope, by a factor of 7.5, i.e. from $30 \mathrm{~nm}$ to $4 \mathrm{~nm}$. According to (1), the optimal $R_{56}$ for the last chicane is by the compression factor smaller than that of the previous chicanes, i.e. it is $10 \mu \mathrm{m}$ (note that the undulator has a non-negligible $R_{56}=3 \mu \mathrm{m}$ which has to be taken into account). In this case the gain per cascade is the same, i.e. it is about 10 in our example for the compressed slice, what is sufficient for saturation. The current in this slice has increased by a factor of 7.5 , and the width of the generated current spike is about $10 \mathrm{~nm} \mathrm{rms}$.

The radiator undulator has 5 -10 periods with the period length of $3 \mathrm{~cm}$. The radiated power is estimated with the help of formula (26) at sub-gigawatt level with the pulse duration about 100 as. The contrast is expected to be high since there is no significant amplification in the uncompressed part of the bunch at $4 \mathrm{~nm}$, and saturation at $30 \mathrm{~nm}$ is not achieved (thus there is no harmonics generation) because there is almost no gain in the last cascade due to reduced $R_{56}$. Pulse energy of spontaneous radiation from the whole bunch within coherent angle is at the level of a few per cent of the attosecond pulse energy. Note that in htis specific example the compression factor could be made larger if a stronger energy modulation by the laser could be achieved. One should also notice that total length of the system is rather small, less than $10 \mathrm{~m}$.

\subsection{LSCA as a source of radiation with a relatively large bandwidth}

FEL radiation has narrow band, typically 0.1-1 \%. For some experiments, however, a relatively large bandwidth is required, up to $10 \%$. In FELs an increase can be achieved by imposing on the electron beam an energy chirp, which is translated into radiation frequency chirp. This approach has technical limits: accelerator has a finite energy acceptance, and it is not always possible to impose required energy chirps on very short bunches. In an LSCA the density modulation is broadband, and the radiation bandwidth (within the central cone of undulator radiation) is given by inverse number of undulator periods. 


\subsection{LSCA driven by a laser-plasma accelerator}

The technology of laser-plasma accelerators progresses well, ${ }^{27}$ a GeV beam is already obtained. ${ }^{28}$ The electron beam with the energy about $200 \mathrm{MeV}$ was sent through the undulator, and spontaneous undulator radiation at $18 \mathrm{~nm}$ wavelength was obtained. ${ }^{29}$ The VUV and X-ray FELs driven by these accelerators are proposed..$^{30,31}$ However, it is not clear at the moment if tight requirements on electron beam parameters and their stability, overall accuracy of the system performance etc., could be achieved in the next years.

Contrary to FELs, the amplification mechanism of LSCA is very robust. For example, it can tolerate large energy chirps. In the case of an FEL the energy chirp parameter is $\lambda h / \rho^{2}$, where $\rho$ is the FEL parameter ${ }^{33}$ defining, in particular, SASE FEL bandwidth. The energy chirp parameter should be small as compared to unity in order to not affect FEL gain. Contrary to that, mechanism of LSCA is broadband, so that "effective $\rho$ " is on the order of one. In other words, in a drift space the influence of the chirp can be always neglected. Of course, if one would like to avoid compression (decompression) in chicanes of LSCA, one should require $h R_{56} \ll 1$. If the $R_{56}$ is chosen according to (10), then the condition for the chirp can be formulated as $h \lambda \ll \sigma_{\gamma} / \gamma$.

In an FEL there are stringent requirements on straightness of the trajectory: the electron beam must overlap with radiation over a long distance. In the case of LSCA one should only require that the angles of the electron orbit should be smaller that $\lambda / \sigma_{\perp}$ what means for the optimal wavelength $\gamma_{z}^{-1}$.

One can speculate (since some important parameters of beams have never been measured) that LSCA could be an interesting alternative to FELs, at least as the first step towards building light sources based on laserplasma accelerators. One of the most important unknown parameters is the slice energy spread (slice size is given by a typical wavelength amplified in LSCA), since the measured value is usually a projected energy spread, dominated by an energy chirp along the bunch. An interesting option would be to use an energy chirp, induced by LSC and wakefields over the whole bunch, ${ }^{30,32}$ for the wavelength compression as discussed in Section 2 . Taking into account the sign of the energy chirp, one should use, for instance doglegs instead of chicanes. One can also consider LSCA as a preamplifier (making sure that it does not saturate) with the final amplification in an FEL.

\section{DISCUSSION}

In this paper we introduced the concept of the Longitudinal Space Charge Amplifier (LSCA) that can operate in VUV and X-ray ranges. Although such an amplifier can not directly compete with FELs in terms of shorter wavelength, higher power, brilliance etc., one can nevertheless find interesting applications for it. In particular, it can be a cheap addition to some existing or planned FEL systems helping to extend operating range towards longer wavelength and to provide the second color for pump-probe experiments. Broadband nature of the amplifier supports production of short (down to few cycles) VUV and X-ray pulses. Bandwidth of the radiation from the undulator of LSCA can be controlled by choosing the number of undulator periods. In particular, one can produce powerful radiation with a relatively large bandwidth what might be difficult in an FEL. Robustness of LSCA makes it an interesting alternative to an FEL in light sources driven by laser-plasma accelerators.

There are many different possibilities that were not considered in this paper and are left for future studies. In particular, we did not study nonlinear harmonic generation, an effect that should occur at the saturation of LSCA. Since amplification mechanism of LSCA is broadband, the bands of harmonics might even partially overlap. The radiation wavelength within the central cone is controlled by tuning the undulator parameter. Also, for a planar undulator there might be a set of odd harmonics on axis.

We have considered in this paper a start up of LSCA from shot noise. However, LSCA can also amplify a coherently seeded density modulation. In this case one needs an undulator and a chicane in front of the first cascade of LSCA. The electron beam gets energy-modulated by a laser beam in the undulator, and in the chicane these energy modulations are converted into coherent density modulations. Particularly interesting might be a seeding in a few-period undulator by attosecond VUV pulses, obtained by high harmonics generation (HHG) in gases by powerful few-cycle lasers. ${ }^{34,35}$ Short few-cycle density modulations can be amplified through LSCA without lengthening, and few-period radiator undulator would produce powerful few-cycle VUV radiation. This option is not available in an FEL amplifier due to a narrow bandwidth. 
It was briefly mentioned in the paper that LSCA can serve as a preamplifier for a SASE FEL. There might be other options, for instance putting LSCA with a short undulator in an optical cavity, thus having, for instance, a regenerative amplifier with a desirable bandwidth. We should also notice here a possibility of using LSCA for some amplification (not necessarily to saturation) of shot noise in light sources based on spontaneous radiation in undulators, for example driven by energy-recovery linacs. In this way one can significantly enhance radiation intensity and brilliance.

Finally, we have to mention that a possibility of a harmful LSC instability at short wavelengths (VUV and soft X-ray) should not be forgotten. Such an instability can develop parasitically in FEL systems (at wavelengths that are much longer than the FEL wavelength) with dispersive elements, such as chicanes in high-gain harmonic generation schemes (especially dangerous can be "fresh bunch" chicanes), achromatic bends for separation of beamlines, chicanes in seeding and self-seeding schemes etc. If LSC instability develops to a significant level of density modulations, strong energy modulations (acting as local energy spread) can be induced in last parts of FELs thus hampering their operation.

\section{ACKNOWLEDGEMENTS}

We would like to thank R. Brinkmann, M. Dohlus, P. Emma, F. Gruener, Z. Huang, A. Mesek, and M. Venturini for useful discussions.

\section{REFERENCES}

1. E.L. Saldin, E.A. Schneidmiller and M.V. Yurkov, Nucl. Instrum. and Methods A483, 516, 2002.

2. E.L. Saldin, E.A. Schneidmiller and M.V. Yurkov, Nucl. Instrum. and Methods A528, 355, 2004

3. Z. Huang et al., Phys. Rev. ST Accel. Beams 7,074401, 2004

4. M. Venturini, Phys. Rev. ST Accel. Beams 11, 034401, 2008

5. D.F. Ratner, A. Chao, and Z. Huang, Proceedings of the FEL2008 Conference, p. 338

6. H. Loos et al., Proceedings of FEL2008 Conference, p. 485

7. S. Wesch et al., Proceedings of FEL2009 Conference, p. 619

8. A. Lumpkin et al., Phys. Rev. ST Accel. Beams 12, 080702, 2009

9. Z. Huang et al., PRST-AB 13, 020703, 2010

10. A.M. Kondratenko, E.L. Saldin, Sov. Phys. Dokl. vol. 24, No. 12 (1979)986; Part. Accelerators 10, 207, 1980.

11. E.L. Saldin, E.A. Schneidmiller and M.V. Yurkov, Nucl. Instrum. and Methods A490, 1, 2002.

12. S. Heifets, G. Stupakov and S. Krinsky, Phys. Rev. ST Accel. Beams 5, 064401, 2002.

13. Z. Huang and K.-J. Kim, Phys. Rev. ST Accel. Beams 5, 074401, 2002.

14. G. Geloni et al., Nucl. Instrum. and Methods A578, 34, 2007.

15. E.L. Saldin, E.A. Schneidmiller and M.V. Yurkov, Nucl. Instrum. and Methods A539, 499, 2005.

16. M. Altarelli et al. (Eds.), XFEL: The European X-Ray Free-Electron Laser. Technical Design Report, Preprint DESY 2006-097, DESY, Hamburg, 2006 (see also http://xfel.desy.de).

17. R. Brinkmann, E.A. Schneidmiller, and M.V. Yurkov, Nucl. Instrum. and Methods A616, 81, 2010.

18. W. Ackermann et al., Nature Photonics 1, 336, 2007.

19. K. Tiedtke et al., New Journal of Physics 11, 023029, 2009.

20. E.L. Saldin, E.A. Schneidmiller, M.V. Yurkov, Optics Communications 212, 377, 2002.

21. E.L. Saldin, E. A. Schneidmiller and M. V. Yurkov, Optics Communications 237, 153, 2004.

22. A.A. Zholents and W.M. Fawley, Phys. Rev. Lett. 92, 224801, 2004.

23. P. Emma, Z. Huang and M. Borland, Proc. of the FEL2004 Conference, p. 333.

24. A.A. Zholents and G. Penn, Phys. Rev. ST Accel. Beams 8, 050704, 2005.

25. E.L. Saldin, E.A. Schneidmiller and M.V. Yurkov, Phys. Rev. ST Accel. Beams 9, 050702, 2006.

26. D. Xiang, Z. Huang, and G. Stupakov, Phys. Rev. ST Accel. Beams 12, 060701, 2009.

27. E. Esarey, C.B. Schroeder, and W.P. Leemans, Reviews of Modern Physics 81, 1229, 2009.

28. W.P. Leemans et al., Nature Phys. 2, 696, 2006. 
29. M. Fuchs et al., Nature Phys. 5, 826, 2009.

30. F. Gruener et al., Appl.Phys. B86, 431, 2007.

31. C.B. Schroeder et al., Proceedings of the FEL2006 Conference, p. 455

32. G. Geloni et al., Nucl.Instrum.Meth. A578, 34, 2007.

33. R. Bonifacio, C. Pellegrini and L.M. Narducci, Opt. Commun. 50, 373, 1984.

34. R. Kienberger et al., Nature 427, 817, 2004.

35. G. Sansone, Science 314, 443, 2006. 